\title{
An image segmentation algorithm for measurement of flotation froth bubble size distributions
}

\begin{abstract}
The bubble size distribution at the froth surface of a flotation cell is closely related to the process condition and performance. The flotation performance can be reasonably predicted through continuous measuring the bubble size distribution by a machine vision system. In this work a new watershed algorithm based on whole and sub-image classification techniques is introduced and successfully validated by several laboratory and industrial scale froth images taken under different process conditions. The results indicate that the developed algorithms, in particular the sub-image classification based segmentation algorithm, can accurately and reliably identify the individual small and large bubbles in the actual froth images, which is often problematic.
\end{abstract}

Keyword: Froth flotation; Image classification; Segmentation; Watershed algorithm 